\section{Avaliação da concordância dos métodos de pesagem direta de alimentos em creches - São Paulo - Brasil}

\section{Measuring agreement in "Direct Food Weighing Methods" in child care centers in São Paulo - Brazil}

\author{
Ana Teresa Rodrigues Cruz \\ Departamento de Nutrição \\ Faculdade de Saúde Pública \\ Universidade de São Paulo \\ Av. Dr. Arnaldo, 715 - $2^{\circ}$ andar - Cerqueira César \\ 01246-090 - São Paulo - SP \\ anateresacruz@aol.com
}

\section{José Maria Pacheco de Souza \\ Departamento de Epidemiologia \\ Faculdade de Saúde Pública \\ Universidade de São Paulo}

\section{Sonia Tucunduva Philippi}

Departamento de Nutrição

Faculdade de Saúde Pública

da Universidade de São Paulo

\section{Auxilio Financeiro}

Fundação de Amparo à Pesquisa do Estado de São Paulo - FAPESP

(Processo no 98/08095-9)

Projeto temático "Nutrição e Saúde: Uma abordagem integrada para avaliação nutricional, desenvolvimento de alimentos para fins especiais e intervenção nutricional"

\section{Resumo}

Nos primeiros anos de vida, é essencial para o crescimento e desenvolvimento da criança uma alimentação adequada. Uma alimentação bem planejada pela creche deve fazer parte das metas do Estado e do município dentro de suas políticas públicas, havendo a necessidade da existência de métodos que avaliem, de forma rápida e simples, o consumo alimentar. Objetivo: Avaliar a concordância dos dados de consumo alimentar pelo "método da pesagem direta de alimentosindividual" e "método da pesagem direta de alimentos-total”. Materiais e Métodos: Os dados foram obtidos em três creches pelos métodos "pesagem direta de alimentos-individual" e "pesagem direta de alimentostotal" servidos às crianças. Quantificou-se a porção em gramas dos alimentos, assim como as sobras alimentares. A comparação foi feita só por gramas (quantidade consumida). Utilizou-se o coeficiente de concordância de acordo com o Modelo de Lin e a metodologia descrita por Bland \& Altman para a representação gráfica na comparação dos métodos. Resultados: A concordância observada na comparação do "Método da pesagem direta de alimentos-individual" com "Método da pesagem direta de alimentos-total" foi de $\rho=0,676$. Conclusão: A comparação entre o "Método da pesagem direta de alimentos-individual" e o "Método da pesagem direta de alimentostotal" nas creches apresenta baixa concordância. Não se recomenda, portanto, a utilização do "Método da pesagem direta de alimentos-total" para avaliar o consumo alimentar das crianças em creches.

Palavras-chave: Consumo de Alimentos. Creche. Alimentação Infantil. 


\section{Abstract}

In the first years of life, children must have a balanced diet in order to ensure their healthy growth and physical development. Therefore, it is important to have quick and simple methods to evaluate food consumption. Objectives: to evaluate the agreement of the information obtained by the "individual direct food weighing method" and by the "total direct food weighing method". Methods: The methods were the "individual direct food weighing method" and "total direct food weighing method" used in every meal served to children in a child day care center so as to collect food intake information. Each food portion served to the children, as well as leftovers, was quantified in grams. The agreement coefficient was used in accordance with LIN's model and BLAND \& ALTMAN's model for the graphic representation of comparison of both direct food-weighing methods. Results: The agreement observed for the comparison of the Direct Weighing Methods was $\rho=0.676$. Conclusion: The comparison between the "individual direct weighing method" and the "total direct weighing method" in the day care center presented low agreement. Using the "total direct weighing method" to evaluate children's food consumption in day care centers is not therefore recommended.

Keywords: Food intake. Child day care centers. Infant nutrition.

\section{Introdução}

A alimentação infantil constitui um dos aspectos fundamentais para a saúde da criança, e é de extrema importância a adoção de práticas alimentares adequadas nos seus primeiros anos de vida ${ }^{1}$. O acompanhamento da situação nutricional das crianças constitui um instrumento de grande importância para aferição das condições de saúde da população infantil ${ }^{2}$, sendo a vigilância nutricional por meio da avaliação do consumo alimentar um dos pontos de estrangulamento na implementação das políticas públicas.

Um dos fatores determinantes das condições de saúde na infância é a qualidade da alimentação recebida. Sendo este um componente importante do atendimento em creches, organizador do cotidiano da instituição, é fundamental que se avalie suas características e o cumprimento das metas ${ }^{3}$.

A análise inicial dos estudos de consumo alimentar em creches mostra uma diversidade razoável entre eles nos aspectos metodológicos, o que dificulta a comparação dos resultados encontrados ${ }^{3}$.

Freqüentemente encontra-se trabalhos de avaliação de consumo alimentar relacionados com a adequação em relação a recomendações nutricionais, sendo pouco relacionados os trabalhos avaliando resultados de métodos para avaliação do consumo alimentar ${ }^{3}$.

De acordo com Dwyer ${ }^{4}$, várias metodologias vêm sendo utilizadas para quantificar a ingestão de alimentos, no sentido de se obter dados válidos, reprodutíveis e comparáveis. Adota-se, neste caso, uma comparação do método teste com aquele mais tradicional ou de referência ${ }^{4,5}$. No entanto, os estudos de ingestão alimentar são alvo de problemas relativos à validade, devido à inexistência de um "padrão ouro" onde a informação mensurada reflita exatamente o que se pretende medir.

A validade e a precisão das medidas obtidas com questionários dietéticos são usualmente estudadas pela avaliação da concordância com as medições de referência que supostamente fornecem o melhor substituto 
para o verdadeiro valor habitual do consumo de cada indivíduo do estudo de validade ${ }^{6}$.

De acordo com Rodrigo \& Bartrina ${ }^{7}$, o "método da pesagem direta de alimentos" é utilizado em situações onde a população estudada não pode pesar, por si mesma, os alimentos e ainda preencher os instrumentos com os dados sobre os alimentos ingeridos. Assim, quando são realizados estudos de consumo alimentar em creches, escolas, hospitais e comunidades rurais com altas taxas de analfabetismo, tal método de investigação pode ser utilizado com a necessária presença de pesquisadores-observadores no trabalho de campo?

O pesquisador-observador deverá estar presente em cada refeição e pesar cada alimento ou preparação oferecida, considerando cada um dos ingredientes. Uma vez terminada a refeição, pesará as sobras. É recomendado que faça uma visita ao local de estudo para poder coletar informações prévias sobre cardápios, refeições, porções, utensílios, formas de preparo, ingredientes utilizados e proporção dos mesmos em cada preparação, quantidade de óleo, sal e temperos? ${ }^{7}$.

O objetivo do presente estudo é avaliar a concordância dos dados de consumo alimentar pelo "método de pesagem direta dos alimentos-individual" e "método de pesagem direta dos alimentos-total”. Está inserido no sub-projeto "Avaliação do Estado Nutricional: Consumo alimentar de crianças das creches do Município de São Paulo", que tem como objetivo geral avaliar o consumo alimentar de crianças atendidas pelas creches do Município de São Paulo e como objetivos específicos: caracterizar a população de acordo com as variáveis sociodemográficas; avaliar o consumo alimentar das crianças em nível domiciliar e institucional em termos de energia, macro e micronutrientes (cálcio, ferro, vitamina A e vitamina C); descrever o perfil e comportamento alimentares das crianças e famílias; elaborar estratégias e medidas para a intervenção nutricional e propor um sistema de vigilância e saúde que integre o Projeto Temático "Nutrição e Saúde: Uma abordagem integrada para avaliação nutricional, desenvolvi- mento de alimentos para fins especiais e intervenção nutricional". Este é conduzido por docentes do Departamento de Nutrição da Faculdade de Saúde Pública da Universidade de São Paulo desde maio de 1999.

\section{Material e Métodos}

A amostra estudada é de 91 crianças de ambos os sexos, entre 1 e 6 anos de idade, em três creches da Prefeitura do Município de São Paulo. A seleção da creche foi por sorteio, incluindo cada um dos sistemas administrativos: direto, indireto e conveniado.

O levantamento de dados foi precedido de um pré-teste, realizado em uma creche com características semelhantes às das demais creches incluídas no Projeto Temático. O pré-teste teve como objetivo aperfeiçoar o instrumento que foi aplicado na amostra de estudo, testar os recursos humanos e os materiais necessários para a execução da parte prática, além de estimar o tempo necessário para o preenchimento dos formulários impressos.

A coleta de dados deste estudo ocorreu no período de outubro a novembro de 1999; por um dos pesquisadores (A.T.R.C). Foram fornecidas pela instituição listagens com dados pessoais (nome, data de nascimento e sexo) das crianças. A idade em anos de cada criança foi calculada pela diferença entre a data de nascimento e a data da entrevista.

O consumo alimentar foi avaliado utilizando-se dois métodos: "método de pesagem direta de alimentos-total” (por meio do qual foi possível estimar a quantidade de alimentos consumida) e o "método de pesagem direta de alimentos-individual

\section{"Método de pesagem direta de alimentos - total"}

Para determinar a quantidade média de alimentos oferecida a cada criança na creche e a quantidade de alimentos consumidos por refeição, de acordo com os seguintes procedimentos:

- determinou-se a quantidade de alimento/preparação em cada refeição, pesan- 
do o utensílio com o alimento/preparação e descontando-se o peso do utensílio;

- pesou-se a quantidade de sobra limpa (quantidade de alimento que foi preparada e não foi servida às crianças);

- determinou-se a quantidade de alimentos consumidos pelos adultos que realizam suas refeições na creche, estimando que um prato composto por arroz, feijão, carne, legumes e verduras, pesando 400 gramas, que foi baseado na pesagem de 3 amostras de pratos servidos para os adultos das creches e também pela parte comestível do exemplo de uma refeição semelhante, de uma dieta com 1600 kcal da pirâmide alimentar proposta por Philippi e col. ${ }^{8}$ (1999). A proporção dos alimentos na refeição foi calculada com base na quantidade de todos os alimentos componentes da refeição das crianças da creche;

- a quantidade consumida (QC) foi calculada mediante a seguinte fórmula:

QC = Quantidade de alimentos preparada (gramas) - [Quantidade de sobra limpa (gramas) + Quantidade consumida pelos adultos (gramas)]

- dividiu-se a quantidade de alimento consumida pelo número de crianças presentes na creche neste dia.

A quantidade de alimento preparada foi obtida pesando-se todos os alimentos preparados no momento que precedeu a refeição.

\section{"Método da pesagem direta dos alimentos - individual"}

Para determinar a quantidade de alimentos oferecida a cada criança na creche e a quantidade de alimento por ela consumido, foram adotados os seguintes procedimentos:

- pesou-se a quantidade de cada alimento oferecido à criança em cada refeição;

- pesou-se a quantidade de sobra de cada criança;

- determinou-se a quantidade de alimentos consumida (em gramas) pela diferença da quantidade oferecida e sobra;
- realizou-se a média do consumo individual para que pudesse ser feita a comparação dos métodos.

Foi estudada a concordância entre a quantidade de alimento consumida pelas crianças segundo o "Método da pesagem direta dos alimentos-individual" e o "Método da pesagem direta dos alimentos-total", utilizando-se o modelo de $\operatorname{Lin}^{9}$ (1989) e o de Bland \& Altman ${ }^{10}$.

De acordo com o modelo de Lin ${ }^{8}$, a correlação entre dois métodos de investigação pode ser representada graficamente por uma linha de 45 graus com origem no 0 dos eixos $\mathrm{x}$ e y. O coeficiente de concordância (r) é obtido para verificar a concordância entre dois os métodos e inclui medidas de acurácia e precisão.

O modelo de Bland \& Altman ${ }^{10}$ (1999) é utilizado para a representação gráfica de concordância entre métodos. O gráfico é composto de uma linha horizontal representando o ponto de concordância entre os métodos, e outra linha representando a diferença de médias. Quanto mais próximos do eixo de concordância estiverem os pontos, maior a concordância entre os métodos.

Os procedimentos estatísticos foram feitos utilizando-se o software Stata (versão 7.0).

\section{Resultados}

Os dados de valores médios de consumo alimentar referem-se a 3 creches, obtidos em 5 refeições durante 3 dias. Como em uma das creches 3 observações não puderam ser realizadas, há 42 observações.

Na Figura 1 estão apresentados os valores médios do consumo alimentar (g) segundo os dois métodos. Utilizando-se o modelo de $\operatorname{Lin}^{9}$, obteve-se a estimativa de $\tilde{n}=0,676$ com intervalo de confiança [0,522 - 0,822].

Adotando-se o procedimento descrito por Bland \& Altman ${ }^{10}$, observa-se na Figura 2 que a diferença de médias é-9,791 e que há uma certa tendência da diferença entre os dois métodos aumentar com o aumento dos valores médios. O desvio padrão é 42,999 e os limites de concordância de [-94,068; $74,485]$. 


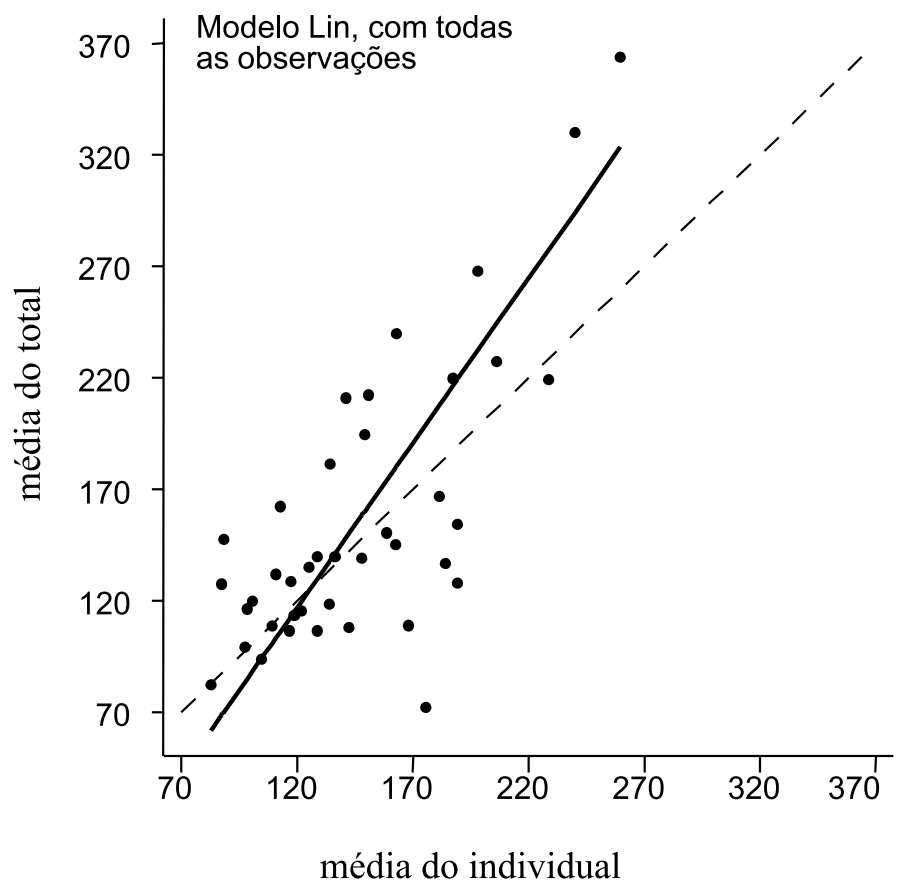

Figura 1 - Valores do consumo alimentar (g) diário de crianças de creche no Município de São Paulo - SP (1999) obtidos através do "Método da pesagem direta dos alimentos individual" e "Método da pesagem direta dos alimentos - total." Modelo de Lin".

Figure 1 - Daily food consumption ( $\mathrm{g}$ ) of children from a child day care center in the city of São Paulo (1999) obtained by the "Individual Direct Food Weighing Method" and "Total Direct Food Weighing Method.'Lin's model'.

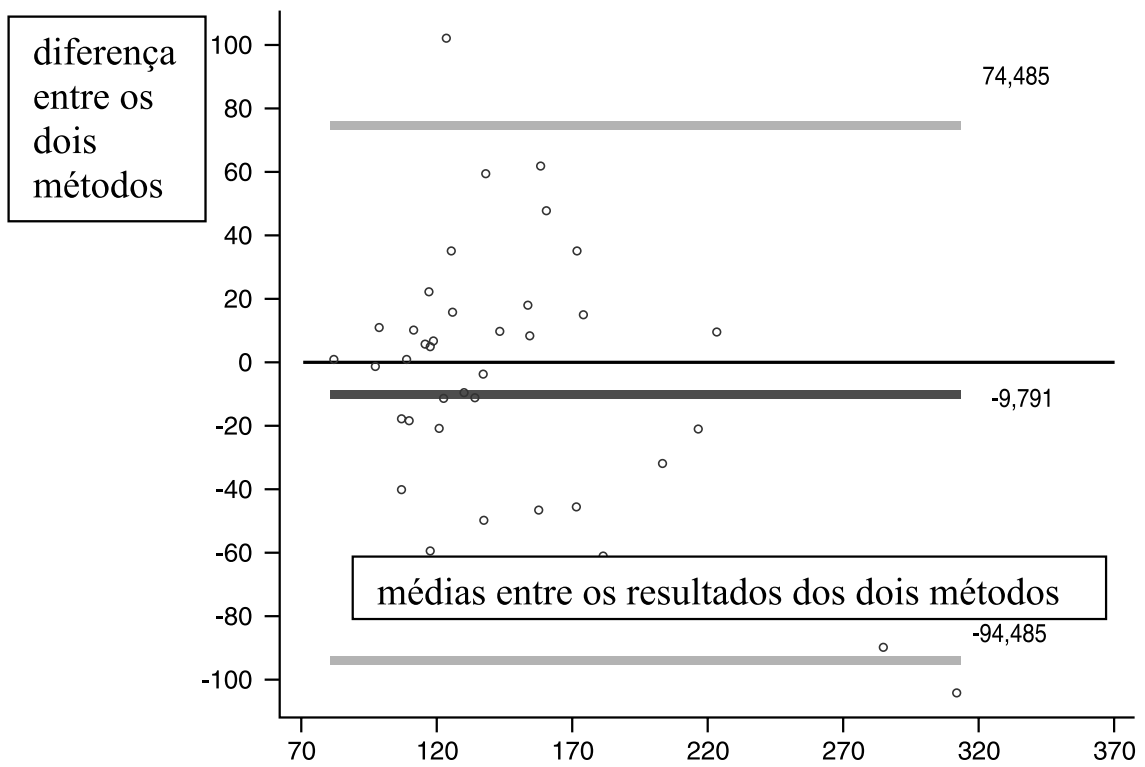

Figura 2 - Valores da diferença de média do consumo alimentar $(\mathrm{g})$ de crianças de creche no Município de São Paulo - SP (1999), utilizando o "Método da pesagem direta dos alimentos individual" e "Método da pesagem direta dos alimentos - total". Modelo de Bland \& Altman ${ }^{10}$. Figure 2 - Difference of average food consumption ( $g$ ) of children from a child day care center in the city of São Paulo - SP (1999), using the «Individual Direct Food Weighing Method» and «Total Direct Food Weighing Method». Bland \& Altman's model ${ }^{10}$. 


\section{Discussão}

No presente estudo o coeficiente de concordância de $\operatorname{LIN}^{9}$ e o modelo de Bland \& Altman ${ }^{10}$ indicaram baixa concordância, não se podendo recomendar o método "pesagem direta de alimentos-total" para avaliar o consumo de alimentos.

Salvo ${ }^{11}$, em um estudo de reprodutibilidade e validade, comparou "Questionário de Freqüência" de consumo de alimento e "Recordatório de 24 horas", utilizando-se de uma população adulta e pré-obesa; o procedimento estatístico utilizado foi a correlação intraclasse e a correlação de Pearson. Em relação à análise de reprodutibilidade da ingestão de nutrientes, os coeficientes de correlação foram de 0,5 a 0,7 , respectivamente.

De acordo com estudo de Pereira ${ }^{12}$, foi comparada a quantidade de fibra alimentar de acordo com o Recordatório de 24 horas e Questionário de Freqüência utilizando-se o modelo de Bland \& Altman ${ }^{10}$. Não houve concordância satisfatória entre os dados de acordo com os métodos utilizados, tendo em vista a amplitude entre os limites de concordância .
Alguns fatos podem interferir na obtenção do consumo real e podem explicar a baixa concordância dos métodos. Alimentos servidos na creche, tais como leite e sucos de frutas, são facilmente derrubados pelas crianças, na maioria das vezes por possuírem pouca habilidade com xícaras e copos. Outro fato observado que dificulta a estimativa individual do consumo é a existência de adultos alimentando-se juntamente com as crianças durante as refeições. A repetição dos alimentos nas refeições é sempre problemática, porque nem sempre a criança consome quantidades iguais, e com relação a alimentos como a carne, somente quando há sobra é oferecida a possibilidade da repetição ${ }^{13}$.

\section{Conclusão}

A comparação entre o "Método da pesagem direta de alimentos-individual" e o "Método da pesagem direta de alimentos-total” nas creches apresenta baixa concordância. Não se recomenda, portanto, a utilização do "Método da pesagem direta de alimentostotal" para avaliar o consumo alimentar das crianças em creches.

\section{Referências}

1. OPAS. Organizacion Panamericana de la Salud. Alimentacion infantil: Bases fisiológicas. Guatemala: OMS/INCAP; 1990

2. Monteiro, CA. Velhos e novos males da saúde no Brasil. São Paulo: Ed. Hucitec; 1995.

3. Zaccarelli EM. Avaliação do estado nutricional de crianças e estrutura de funcionamento de creches no município de São Paulo [dissertação de mestrado]. São Paulo: PRONUT/USP; 2001.

4. Dwyer J. Dietary Assessment. In: Shils ME, Olson JA, Shike M, Ross AC. Modern Nutrition in health and disease. Baltimore: Lippincott Willians \& Wilkins; 1999. p. 937-59.

5. Margetts BM, Nelson M. Design concepts in nutritional epidemiology. New York: Oxford University Press; 1991.
6. Kaaks R, Riboli E, Estève J et al. Estimating the accuracy of dietary questionnaire assessments: validation in terms of structural equation models. Stat Med 1994; 13: 127-42.

7. Rodrigo CPS, Bartrina JA. Diário o registro dietético: métodos de doble pesada. In: Majem LS, Bartrina JÁ, Verdú JM. Nutricion y salud publica; métodos, bases científicas y aplicaciones. Barcelona: Masson; 1995. p. 107-19.

8. Philippi ST, Latterza AR, Cruz ATR, Ribeiro LC. A Pirâmide alimentar adaptada: guia para escolha dos alimentos. Revista de Nutrição da PUCCAMP 1999; 12(1): $65-80$

9. Lin, LI. A concordance correlation coefficient to evaluate reproducibility. Biometrics 1989; 45(1): 25568. 
10. Bland JM, Altman DG. Measuring agreement in method comparison studies. Stat Methods Med Res 1999; 8(2): 135-60.

11. Salvo VLMA. Construção de um questionário de Freqüência de Consumo de alimentos para adultos com excesso de peso. Estudo da reprodutibilidade e validade indireta [dissertação de mestrado]. São Paulo: Escola Paulista de Medicina; 2000.
12. Pereira AC. Ingestão de fibra alimentar por crianças com e sem constipação funcional: Avaliação por 2 inquéritos alimentares e 2 tabelas de composição alimentar [dissertação de mestrado]. Botucatu: Faculdade de Medicina de Botucatu - UNESP; 1999.

13. Ctenas MLD, Vitolo MR. Crescendo com saúde: o guia do crescimento da criança. São Paulo: C2 ed.; 1999.

Recebido em: 23/04/2002

Versão final reapresentada em: 15/10/2002

Aprovação em: 06/06/2003 